\title{
THE RANGE OF AN $o$-WEAKLY COMPACT MAPPING
}

\author{
P. G. DODDS
}

(Received 11 May 1984)

Communicated by J. H. Chabrowski

\begin{abstract}
It is shown that a weakly compact convex set in a locally convex space is a zonoform if and only if it is the order continuous image of an order interval in a Dedekind complete Riesz space. While this result implies the Kluvanek characterization of the range of a vector measure, the techniques of the present paper are purely order theoretic.
\end{abstract}

1980 Mathematics subject classification (Amer. Math. Soc.): primary 46 A 40; secondary 28 B 05,28 C 05 .

\section{Introduction}

Let $A$ be a linear map from an Archimedean Riesz space $L$ to a locally convex space $X$ which maps order intervals of $L$ to bounded subsets of $X$. In this note, it is shown that $A$ maps each order interval of $L$ to a subset of $X$ whose closure in the weak completion of $X$ is a zonoform. It follows, in particular, that if $A$ maps order intervals of $L$ to relatively weakly compact subsets of $X$, then $A$ maps each order interval to a subset of $X$ whose closure is a zonoform in $X$. This result contains as a special case (and, indeed, is strongly motivated by) the characterization of the range of a vector measure given by Kluvanek [6], [7]. The methods of Kluvánek are based on his theory of closed vector measures and integral representation. The present approach is, however, based entirely on the duality theory of Riesz spaces and not only places the results of Kluvánek in quite general setting but shows quite transparently that certain geometric properties of the range of a

(c) 1985 Australian Mathematical Society $0263-6115 / 85 \$ A 2.00+0.00$ 
vector measure are direct consequences of the order structure of the domain of the map and its relation to the order structure in the space of conical measures on the range.

It is convenient to refer to the monograph [11] for basic information concerning the theory of Riesz spaces. As our motivation is drawn from the theory of vector measures, we refer to the monographs [3], [8] for general information concerning the basic elements of the theory of vector measures, although we remark that no explicit use of this theory will be made. For information concerning zonoforms, we refer to [2].

\section{Preliminary results}

A real linear functional on an Archimedean Riesz space $L$ is called order bounded if and only if it maps order intervals to bounded sets. The collection of all order bounded linear functionals on $L$ is denoted $L^{-}$and with respect to the order induced by the positive cone of $L$, it is well known that $L^{\sim}$ is a Dedekind complete Riesz space. If $\phi \in L^{\sim}$, then $\phi$ is called a normal integral on $L$ if and only if whenever $\left\{f_{\tau}\right\} \subseteq L$ and $f_{\tau} \downarrow_{\tau} 0$ in $L$ it follows that $\inf _{\tau}\left|\phi\left(f_{\tau}\right)\right|=0$. The collection of normal integrals on $L$ will be denoted ${L_{n}}_{n}$; the normal integrals on $L$ form a band in $\tilde{L}$. For these results, the reader is referred to [10].

We use the following notation: if $0 \leqslant f \in L$, then $[0, f]$ denotes the order interval $\{g \in L: 0 \leqslant g \leqslant f\}$. The element $0 \leqslant e \in L$ is said to be a strong unit for $L$, if for every $f \in L$, there exists a natural number $n$ such that $f \in[0, n e]$.

Let $L$ be an Archimedean Riesz space and $\Gamma$ a sublattice of the order dual $L^{\tilde{}}$. Let $j: L \rightarrow \Gamma^{\sim}$ be the natural mapping defined by setting $j(f)(\phi)=\phi(f)$ for $f \in L$ and $\phi \in \Gamma$.

Proposition 1. Suppose that the Riesz space $L$ has a strong unit $e$ and $\Gamma$ is a separating Riesz subspace of $L^{\sim}$. The following statements are equivalent.

(i) The order interval $[0, e]$ is $\sigma(L, \Gamma)$ compact.

(ii) $[0, j(e)]=j([0, e])$.

(iii) $L$ is Dedekind complete and $\Gamma$ consists of normal integrals.

Before proceeding to the proof of the Proposition we state separately a Lemma which shall be needed subsequently.

LEMMA 1. If $I$ is the ideal generated by $L$ in $\Gamma^{\sim}$, then $j([0, e])$ is $\sigma(I, \Gamma)$ dense in $[0, j(e)]$.

Proof. Suppose there exists $u \in[0, j(e)] \subseteq I$ such that $u$ does not belong to the $\sigma(I, \Gamma)$ closure of $j([0, e])$. By the separation theorem, there exists an element 
$\phi \in \Gamma$ such that $u(\phi)>\sup \{\phi(f): f \in L, 0 \leqslant f \leqslant e\}=\phi^{+}(e)$. However, $u(\phi) \leqslant$ $u\left(\phi^{+}\right) \leqslant j(e)\left(\phi^{+}\right)=\phi^{+}(e)$ and this is clearly a contradiction.

Proof of the Proposition. The implication (i) $\Rightarrow$ (ii) is clear from the preceding Lemma 1 and the $\sigma(L, \Gamma)$ to $\sigma(I, \Gamma)$ continuity of the map $j$.

(ii) $\Rightarrow$ (iii). Suppose that $0 \leqslant f_{\tau} \uparrow_{\tau} \leqslant f$ with $f_{\tau}, f \in L$. For each $0 \leqslant \psi \in \Gamma$, define $\phi(\psi)=\sup _{\tau} \psi\left(f_{\tau}\right)$. It is not difficult to see that $\pi$ extends to a positive element of $\Gamma^{\sim}$ which is majorized by some multiple of $j(e)$. From (ii), it follows that there exists an element $0 \leqslant g \in L$ such that $\psi(g)=\sup _{\tau} \psi\left(f_{\tau}\right)$ for all $0 \leqslant \psi \in \Gamma$. Since $\Gamma$ is a separating sublattice, it follows that $g=\sup _{\tau} f_{\tau}$ holds in $L$. An exactly similar argument shows that $\Gamma$ consists of normal integrals on $L$.

To see that (iii) $\Rightarrow$ (i), suppose that $L$ is Dedekind complete and that $\Gamma$ consists of normal integrals. It is a well known theorem of $\mathrm{H}$. Nakano that order intervals in $L$ are $\sigma\left(L, L_{n}{ }^{-}\right)$compact; consequently $[0, e]$ is compact for the weaker $\sigma(L, \Gamma)$ topology.

We remark that Proposition 1 contains Lemma 1 of [5] as a special case. See also [8], Theorem 5.2. The importance of Proposition 1 lies in that it can be viewed as an abstract basis for several well known characterizations of localizable measure spaces due to Segal [12, Theorems 3.4 and 5.1].

Proposition 2. (i) Let $L$ be a Riesz space and $K \subseteq L$ a linear subspace. If $\Gamma$ is the Riesz subspace generated by $K$ in $L$, then $f \in \Gamma$ if and only if there exist elements $f_{1}, \ldots, f_{k}, f_{k+1}, \ldots, f_{l}$ of $K$ such that

$$
f=\bigvee_{1 \leqslant i \leqslant k} f_{i}-\underset{k+1 \leqslant i \leqslant l}{\bigvee} f_{i}
$$

(ii) Let $K, K_{0}$ be linear subspaces of the Riesz spaces $L, L_{0}$ and let $\Gamma, \Gamma_{0}$ be the corresponding Riesz subspaces generated by $K, K_{0}$. If $\varphi: K \rightarrow K_{0}$ is a linear map, then $\varphi$ extends to a (necessarily unique) Riesz homomorphism $\Phi$ of $\Gamma$ into $\Gamma_{0}$ if and only if whenever $f_{1}, \ldots, f_{k}, g_{1}, \ldots, g_{l}$ are elements of $K$ such that

$$
\underset{1 \leqslant i \leqslant k}{\bigvee} f_{i}=\bigvee_{1 \leqslant j \leqslant l} g_{j}
$$

holds in $L$, it follows that

$$
\underset{1 \leqslant i \leqslant k}{\bigvee} \varphi\left(f_{i}\right)=\underset{1 \leqslant j \leqslant l}{\bigvee} \varphi\left(g_{j}\right)
$$

holds in $L_{0}$. If $\varphi$ is surjective, then so is $\Phi$.

The proof of the preceding proposition involves only routine manipulations of standard vector lattice identities and the details are accordingly omitted.

The proof of the following proposition was suggested by A. R. Schep. 
Proposition 3. Let $L$ be a Riesz space. Let $\phi_{i}, \psi_{j}, 1 \leqslant i \leqslant l, 1 \leqslant j \leqslant k$, be elements of the order dual $L^{-}$and suppose that

$$
\underset{1 \leqslant i \leqslant l}{\bigvee}\left(\phi_{i}(f)\right)=\bigvee_{1 \leqslant j \leqslant k}\left(\psi_{j}(f)\right)
$$

for every $0 \leqslant f \in L$. Then

$$
\bigvee_{1 \leqslant i \leqslant l} \phi_{i}=\bigvee_{1 \leqslant j \leqslant k} \psi_{j}
$$

holds in $L^{\sim}$.

Proof. Let $\phi=\bigvee_{1 \leqslant i \leqslant l} \phi_{i}$ and let $\psi=\bigvee_{1 \leqslant j \leqslant k} \psi_{j}$. If $0 \leqslant f \in L$, then $\phi(f) \geqslant$ $V_{1 \leqslant i \leqslant l}\left(\phi_{i}(f)\right)=\bigvee_{1 \leqslant j \leqslant k}\left(\psi_{j}(f)\right) \geqslant \psi_{j}(f)$ for $1 \leqslant j \leqslant k$. Consequently $\phi \geqslant$ $\vee_{1 \leqslant j \leqslant k} \psi_{j}=\psi$. By symmetry, it follows that $\phi=\psi$ and the proof is complete.

\section{A Riesz homomorphism and its dual}

In this section, we show that a linear map from a Riesz space $L$ to a locally convex space $X$ induces a Riesz homomorphism which, together with its dual, relates the order structure of $L$ to the order structure of the space of conical measures on $X$. Some preliminary remarks follow.

In the sequel, $L$ always denotes an Archimedean Riesz space with strong unit $e, X$ a locally convex topological vector space and $A: L \rightarrow X$ a linear mapping which maps order intervals in $L$ to bounded subsets of $X$. By $\Gamma$ will be denoted the Riesz subspace in the order dual $L^{\tilde{y}}$ generated by the order bounded functionals $\left\{x^{\prime} \circ A, x^{\prime} \in X^{\prime}\right\}$. Here $X^{\prime}$ denotes the topological dual space of $X$. If $N=\{f \in L:|\phi|(f|f|)=0$ for all $\phi \in \Gamma\}$, then $N$ is an order ideal in $\Gamma$. Observe that $N$ is a band in $L$ if each element of $\Gamma$ is a normal integral on $L$. By passing to the Riesz quotient space $L / N$, it may, and will, be assumed that $\Gamma$ separates the points of $L$. We remark that if $L$ is Dedekind complete and $\Gamma$ consists of normal integrals, then $L / N$ is again Dedekind complete and the functionals induced on $L / N$ by the elements of $\Gamma$ are again normal integrals.

The Riesz subspace of $\mathbf{R}^{X}$ generated by the elements of $X^{\prime}$ with respect to the pointwise order on $X$ will be denoted by $h(X)$. The order structure of $h(X)$ is related to that of $L$ as follows.

TheOREM 1. There exists a unique surjective Riesz homomorphism $\Phi: h(X) \mapsto \Gamma$ such that $\Phi\left(x^{\prime}\right)=x^{\prime} \circ A$ for each $x^{\prime} \in X^{\prime}$. 
Proof. Consider $X^{\prime}$ as a linear subspace of the Riesz space $\mathbb{R}^{X}$ and let $\varphi$ be the linear mapping $x^{\prime} \mapsto x^{\prime} \circ A, x^{\prime} \in X^{\prime}$. The range of $\varphi$ generates the Riesz subspace $\Gamma$. To show that $\varphi$ extends to a Riesz homomorphism of $h(X)$ onto $\Gamma$, it suffices, by Proposition 2, to show that whenever $x_{1}^{\prime}, \ldots, x_{k}^{\prime}, y_{1}^{\prime}, \ldots, y_{l}^{\prime}$ are elements of $X^{\prime}$ such that

$$
\underset{1 \leqslant i \leqslant k}{\bigvee} x_{i}^{\prime}=\bigvee_{1 \leqslant j \leqslant l} y_{j}^{\prime}
$$

it follows that $\bigvee_{1 \leqslant i \leqslant k} x_{i}^{\prime} \circ A=\bigvee_{1 \leqslant j \leqslant l} y_{j}^{\prime} \circ A$. However, (1) asserts that $\bigvee_{1 \leqslant i \leqslant k}\left(x_{i}^{\prime} \circ A(f)\right)=\bigvee_{1 \leqslant j \leqslant k}\left(y_{j}^{\prime} \circ A(f)\right)$ for each $f \in L$. The assertion of the Theorem now follows from Proposition 3.

We mention several immediate consequences of Theorem 1. If $\mathscr{S}$ is a $\sigma$-algebra of subsets of some point set, denote by c.a.( $\mathscr{S})$ the band of (real) countably-additive measures on $\mathscr{S}$. If $m: \mathscr{S} \rightarrow X$ is a vector measure, then it is shown in Theorem 1 of [6], via the Radon-Nikodym theorem, that the vector measure $m$ induces a unique Riesz homomorphism $\Phi_{m}: h(X) \rightarrow$ c.a. $(\mathscr{S})$ such that $\Phi_{m}\left(x^{\prime}\right)=x^{\prime} \circ m$, for each $x^{\prime} \in X^{\prime}$. That this result is an immediate consequence of Theorem 1 above may be seen by taking $L$ to be the Riesz space of all step-functions based on $\mathscr{S}, A$ to be the integration map on $L$ induced by $m$ and $\Gamma$ to be the band c.a.( $\mathscr{S})$.

In a similar manner, Lemma 7 of [7] also follows directly from Theorem 1 above. We leave the details to the interested reader.

Let $I$ be the order ideal in $\Gamma^{\sim}$ generated by $L$ and let $\Phi: h(X) \rightarrow \Gamma$ be the Riesz homomorphism whose existence is shown by the preceding theorem. Denote by $M(X)$ the order dual of the Riesz space $h(X)$. Elements of the positive cone of $M(X)$ are called conical measures on $X$ and these are denoted by $M^{+}(X)$. For basic facts concerning conical measures, we refer to [2, Sections $30,38,40]$.

Define now the linear map $\tilde{\tilde{A}}: I \rightarrow M(X)$ by setting $\tilde{\tilde{A}}(\phi)=\phi \circ \Phi$ for each $\phi \in I$. Let $j: L \rightarrow I$ be the natural map defined by setting $j(f)(\psi)=\psi(f)$ for each $\psi \in \Gamma$ and $f \in L$. The map $j$ is linear and order preserving. Of interest is the order interval in $M^{+}(X)$ generated by the conical measure $\tilde{\tilde{A}}(j(e))$. For the case that $A$ is the integration map induced by a vector measure $m, \tilde{\tilde{A}}(j(e))$ is precisely the conical measure $\Delta(m)$ introduced in [6].

THEOREM 2. $\tilde{\tilde{A}}([0, j(e)])=[0, \tilde{\tilde{A}}(j(e))]$.

We observe that the map $\tilde{\tilde{A}}$ is the restriction to $I$ of the Riesz space adjoint $\Phi^{\sim}$ of $\Phi$ where $\Phi^{\sim}: \Gamma^{\sim} \rightarrow h(X)^{\tilde{}}=M(X)$. Thus Theorem 2 is an immediate consequence of the well known result that if $L$ and $M$ are Archimedean Riesz 
spaces with separating order duals, then a positive linear map $T$ from $L$ to $M$ is a Riesz homomorphism if and only if the Riesz space adjoint of $T$ is order interval preserving. See for example [9, Theorem 5.15].

\section{Weakly normal mappings}

We continue with the notation and assumptions of the preceding section. The linear map $A: L \rightarrow X$ will be called weakly normal if and only if $\left\{f_{\tau}\right\} \subseteq L$ and $f_{\tau} \downarrow{ }_{\tau} 0$ imply that $A f_{\tau} \rightarrow 0$ weakly in $X$. If $0 \leqslant u \in M^{+}(X)$, then an element $x \in X$ is called the resultant of $u$ if $u\left(x^{\prime}\right)=x^{\prime}(x)$ for all $x^{\prime} \in X^{\prime}$. The resultant of $u$, if it exists, is unique and is denoted $r(u)$. If $0 \leqslant u \in M^{+}(X)$ and if $r(v)$ exists for every $v \in M^{+}(X)$ with $0 \leqslant v \leqslant u$, then the set $r([0, u])$ is called a zonoform. A zonoform in $X$ is convex and weakly compact.

THEOREM 3. Let $L$ be Dedekind complete and let $A: L \rightarrow X$ be weakly normal. If $0 \leqslant v \in M^{+}(X)$ and $0 \leqslant v \leqslant \tilde{\tilde{A}}(j(e))$, then $v$ has a resultant in $X$ and $A([0, e])=$ $r([0, \tilde{\tilde{A}}(j(e))])$.

Proof. By the implication (iii) $\Rightarrow$ (ii) of Proposition 1 and by Theorem 2, it suffices to show that if $f \in L$ and $0 \leqslant f \leqslant e$, then $r(\tilde{\tilde{A}}(j(f)))=A f$. Let $x^{\prime} \in X^{\prime}$ and observe that $\tilde{\tilde{A}}(j(f))\left(x^{\prime}\right)=j(f) \circ \Phi\left(x^{\prime}\right)(f)=x^{\prime} \circ A(f)=x^{\prime}(A f)$. By This, the theorem is proved.

The notion of closed vector measure was introduced in [5]. It is shown in [8] that the integration map induced by a closed vector measure is a weakly normal map defined on a Dedekind complete Riesz space.

The above theorem therefore implies that the closed convex hull of the range of a closed vector measure $m: \mathscr{S} \rightarrow X$ is a zonoform, is weakly compact and is precisely the range of the induced integration map restricted to the set of

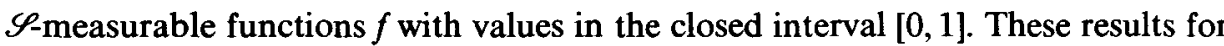
vector measures were first established in [5, Theorem 2] and in [6, Theorem 2 and Theorem 4]. We now show that each zonoform in $X$ is the image of an order interval in a Dedekind complete Riesz space under a weakly normal mapping. It is then not difficult to see directly, by passing to an appropriate Stone space, that each zonoform in $X$ is the closed convex hull of a closed vector measure, so that our methods yield [5, Theorem 5]. 
Theorem 4. Let $X$ be a locally convex topological space. Let $u \in M^{+}(X)$ and suppose that each element of the order interval $[0, u] \subseteq M^{+}(X)$ has resultant in $X$. There exists a Dedekind complete Riesz space $L$ with strong order unit $e$ and a weakly normal linear mapping $A: L \rightarrow X$ such that $A([0, e])=r([0, u])$.

Proof. The Riesz space $M(X)$, being the order dual of the Riesz space $h(X)$, is Dedekind complete. Let $L$ be the order ideal generated by $u$ in $M(X)$ and let $A$ be the restriction of the resultant map to $L$. Suppose that $\left\{u_{\tau}\right\} \subseteq L$ and $u_{\tau} \downarrow_{\tau} 0$ in $L$. Then also $u_{\tau} \downarrow \downarrow_{\tau} 0$ in $M^{+}(X)$. Since each element of $h(X)$ defines a normal integral on $M(X)$, it follows that $u_{\tau}\left(x^{\prime}\right) \rightarrow 0$ for each $x^{\prime} \in X^{\prime}$. Thus $x^{\prime}\left(r\left(u_{\tau}\right)\right)=$ $u_{\tau}\left(x^{\prime}\right) \rightarrow 0$ for each $x^{\prime} \in X^{\prime}$. This says that $A u_{\tau} \rightarrow 0$ weakly in $X$ and the proof is complete.

The weakly normal mappings have a number of strong properties which are indicated in Proposition 4 below.

The map $A: L \rightarrow X$ is called normal if and only if $\left\{f_{\tau}\right\} \subseteq L$ and $f_{\tau} \downarrow_{\tau} 0$ imply $A f_{\tau} \rightarrow 0$ in $X$. The Riesz semi-norm $\rho$ on $L$ is called normal if and only if $\left\{f_{\tau}\right\} \subseteq L$ and $f_{\tau} \downarrow_{\tau} 0$ imply $\rho\left(f_{\tau}\right) \downarrow_{\tau} 0$. If $H \subseteq X^{\prime}$ is equicontinuous, let $\rho_{H}$ be the Riesz semi-norm on $L$ defined via $\rho_{H}(f)=\sup \left\{\left|x^{\prime} \circ A\right|(|f|): x^{\prime} \in H\right\}$ for each $f \in L$. Denote by $T$ the locally solid Hausdorff topology on $L$ defined by the collection of Riesz semi-norms $\left\{\rho_{H}\right\}$.

Proposition 4. If $L$ is Dedekind complete and if $A: L \rightarrow X$ is weakly normal, then

(i) $A([0, e])$ is $\sigma\left(X, X^{\prime}\right)$ compact,

(ii) whenever $H \subseteq X^{\prime}$ is equicontinuous, the Riesz semi-norm $\rho_{H}$ is normal,

(iii) $A$ is normal,

(iv) the order interval $[0, e]$ is T-Complete.

Proof. (i) is an immediate consequence of Theorem 4 above.

(ii) It is a consequence of the weak normality of $A$, the well-known OrliczPettis lemma and part (i) above that, if $\left\{f_{n}\right\} \subseteq L$ is a sequence and if $0 \leqslant f_{n} \uparrow f \in L$, then $A f_{n} \rightarrow 0$ in $X$. If $H \subseteq X^{\prime}$ is equicontinuous, then the normality of the Riesz semi-norm $\rho_{H}$ is a consequence of $[4$, Theorem A].

Statement (iii) is a simple consequence of (ii) and the $T$-completeness of the order interval $[0, e]$ follows immediately from (ii) and the Nakano completeness theorem [1, Theorem 13.1].

It is to be remarked that statement (i) of the preceding proposition may be deduced directly from the weak normality of $A$ and the fact that order intervals in $L$ are $\sigma\left(L, L_{n}{ }^{2}\right)$ compact. 
We now show that if $A: L \rightarrow X$ is arbitrary, then $A$ always has a range-preserving extension to a weakly normal map on some Dedekind complete Riesz space containing $L$, with values in the weak completion of $X$. This is the principal result of the paper and can be viewed as the abstract core of the notion of closure of a vector measure introduced in [5]. For the special case that $A$ is the integration map induced by an arbitrary vector measure $m$, we obtain that the closed convex hull of the range of $m$ is a zonoform, which is [6, Theorem 4]. It is worth remarking however, that Theorem 5 below also applies directly to bounded, additive (rather than countably additive) mappings defined on an algebra of sets. Moreover, our method shows clearly that the purely geometric properties of the range of such mappings do not depend on the representation theory developed in [7], which is, of course, of independent interest.

Our notation in the following is that established in earlier sections. If $E$ is a subset of $X$, we denote by $\bar{E}$ the closure of $E$ in $X$.

ThEOREM 5. Let $\hat{X}$ be the weak completion of X. The map $\hat{A}=r \circ \tilde{\tilde{A}}$ is a weakly normal linear mapping of the Dedekind complete Riesz space $I$ into $\hat{X}$ such that $\hat{A} \circ j=A$. Moreover, $\hat{A}([0, j(e)])$ is precisely the $\sigma\left(\hat{X}, X^{\prime}\right)$ closure of $A([0, e])$ in $\hat{X}$. Consequently, if $A([0, e])$ is relatively weakly compact in $X$, then $\overline{A([0, e])}$ is a zonoform.

Proof. By [2, Proposition 30.7] the map $\hat{A}=r \circ \tilde{\tilde{A}}$ is defined on $I$ and takes values in $\hat{X}$. Suppose that $\left\{f_{\tau}\right\} \subseteq I$ and that $f_{\tau} \downarrow_{\tau} 0$ in $I$. This means that $\phi\left(f_{\tau}\right) \rightarrow 0$ for every $\phi \in \Gamma$. Hence $\Phi(h)\left(f_{\tau}\right) \rightarrow 0$ for every $h \in h(X)$ and so $\tilde{\tilde{A}}\left(f_{\tau}\right)(h)=f_{\tau} \circ \Phi(h) \rightarrow 0$ for every $h \in h(X)$. It follows that $x^{\prime}\left(\hat{A} f_{\tau}\right)=$ $\left.x^{\prime} \circ \tilde{A}\left(f_{\tau}\right)\right)=\tilde{\tilde{A}}\left(f_{\tau}\right)\left(x^{\prime}\right) \rightarrow 0$ and so $\hat{A}$ is weakly normal. From the definition of $\hat{A}$, it is clear that $A \circ j=\hat{A} \circ j$, that $\tilde{A}([0, j(e)])$ is $\sigma\left(\hat{X}, X^{\prime}\right)$ compact and that $\overline{A([0, e])} \subseteq \hat{A}([0, j(e)])$. That $\overline{A([0, e])}=\hat{A}([0, j(e)]$ is now a consequence of Lemma 1.

In conclusion, we mention the following consequence.

COROLLARY. If $X$ is weakly complete, then $A([0, e])$ is relatively weakly compact.

\section{References}

[1] C. D. Aliprantis and O. Burkinshaw, Locally solid Riesz spaces, (Academic Press, New York, San Francisco, London, 1978).

[2] G. Choquet, Lectures on analysis, Eds. Marsden, Lance and Gelbart, (W. A. Benjamin, New York, Amsterdam, 1969).

[3] J. Diestel and J. J. Uhl, Jr., 'Vector measures', Math. Surveys 15 (Amer. Math. Soc., 1977). 
[4] P. G. Dodds, 'o-weakly compact mappings of Riesz spaces', Trans. Amer. Math. Soc. 214 (1975), 389-402.

[5] I. Kluvanek, 'The range of a vector-valued measure', Math. Systems Theory 7 (1973), 44-54.

[6] I. Kluvanek, 'Characterization of the closed convex hull of the range of a vector-valued measure', J. Funct. Anal. 21 (1976), 316-329.

[7] I. Kluvanek, 'Conical measures and vector measures', Ann. Inst. Fourier (Grenoble) 271 (1977), 83-105.

[8] I. Kluvánek and G. Knowles, Vector measures and control systems (North-Holland Publishing Co., Amsterdam, 1975).

[9] W. A. J. Luxemburg, Some aspects of the theory of Riesz spaces (The University of Arkansas Lecture Notes in Mathematics, \# 4, Fayetteville, 1979).

[10] W. A. J. Luxemberg and A. C. Zaanen, 'Notes on Banach function spaces VI-XIII', Nederl. Akad. Wetensch. Proc. Ser. A 66 (1963), 251-263, 496-504, 655-681; 67 (1964), 104-119, 360-376, 493-518, 519-543.

[11] W. A. J. Luxemberg and A. C. Zaanen, Riesz spaces (North-Holland, 1971).

[12] I. E. Segal, 'Equivalences of measure spaces', Amer. J. Math. 73 (1951), 275-313.

School of Mathematical Sciences

The Flinders University of South Australia

Bedford Park, S.A. 5042

Australia 